\title{
THE SWALLOWTAIL AND BUTTERFLY CUSPOIDS AND THEIR APPLICATION IN THE INITIAL POST-BUCKLING OF SINGLE-MODE STRUCTURAL SYSTEMS*
}

\author{
BY \\ DAVID HUI AND JORN S. HANSEN \\ University of Toronto
}

\begin{abstract}
This paper investigates the swallowtail and butterfly catastrophes from the point of view which is applicable in the theory of elastic stability. Thus, the results are concerned with the various forms of these instabilities as well as the determination of the critical load surfaces which are of engineering significance. It is demonstrated that the results are applicable to an axially loaded beam resting on a nonlinear elastic foundation.
\end{abstract}

Introduction. Catastrophe theory [1] and the theory of elastic stability [2, 3, 4] are known to be similar in nature. For example, Thompson and Hunt [5] showed that imperfection sensitivity in the anticlinal point of bifurcation results in an elliptic umbilic catastrophe. Further, they showed that the stability analysis of a two-mode axially loaded stiffened flat plate could be classified as a hyperbolic umbilic catastrophe. In addition, the parabolic umbilic and its application to a two-mode pressurized spherical shell and to a two-mode uniformly compressed plate on a nonlinear elastic foundation were examined by Hui and Hansen [6,7]. For single-mode systems, the catastrophes which have been considered are the fold, cusp, swallowtail and butterfly cuspoids. The fold model occurs when the cubic term of the potential energy is non-zero. It arises, for example, in the single-mode overall buckling of a wide integrally stiffened flat plate (where the stringers are closely spaced so that overall buckling occurs first) under axial compression [8] and in the Cox buckling problem [2]. The cusp model is found in both the single local mode and the single overall mode buckling of axially stiffened cylindrical shells under compressive loads $[9,10,11]$, oval cylindrical shells under axial compression [12], compressed sandwich cylindrical panels [13] as well as in the initial post-buckling analysis of a spherical cap under a concentrated or an axisymmetric distributed load [14, 15]. The reason for the present investigation is that all of the above-mentioned elementary catastrophes have been investigated and applied to structural instability problems except the swallowtail and the butterfly cuspoids. Thus, it is of interest to investigate the implications of these remaining catastrophes and to determine the role they play in the theory of elastic stability.

In this paper, the swallowtail and butterfly cuspoids are unfolded and analyzed in terms of the theory of elastic stability. They are solved in the most general form so that

* Received June 21, 1979. 
the results are applicable to any system where the potential energy expression falls into their standard forms. Previous work $[16,17,18]$ has been done on these models; however, it was not specifically toward the theory of elastic stability. In particular, the equilibrium paths of the present work illustrate important physical insight and as a result emphasize the distinction between primary and secondary critical surfaces. The swallowtail and butterfly cuspoids are in general applicable to any cusp catastrophe (including all the aforementioned examples) when the quartic term of the potential energy is either zero or sufficiently small. Therefore, in order to demonstrate the general results, a stability analysis of a beam resting on a nonlinear elastic foundation is presented in the final section. This analysis is based on a Koiter-style approach [2].

Classification of single-mode systems. The most general form of the potential energy of a single-mode system expanded about the classical critical load of the perfect system is

$$
\begin{aligned}
P E= & {\left[A_{2}^{\prime}\left(\lambda-\lambda_{c l}\right)+\frac{1}{2 !} A_{2}^{\prime \prime}\left(\lambda-\lambda_{c l}\right)^{2}+\cdots\right] \xi^{2}+\left[A_{3}+A_{3}^{\prime}\left(\lambda-\lambda_{c l}\right)+\cdots\right] \xi^{3} } \\
& +\left[A_{4}+A_{4}^{\prime}\left(\lambda-\lambda_{c l}\right)+\cdots\right] \xi^{4}+\left[A_{5}+A_{5}^{\prime}\left(\lambda-\lambda_{c l}\right)+\cdots\right] \xi^{5} \\
& +\left[A_{6}+A_{6}^{\prime}\left(\lambda-\lambda_{c l}\right)+\cdots\right] \xi^{6}+\cdots \\
& +\left[\varepsilon_{1} B_{1}+\varepsilon_{1} B_{1}^{\prime}\left(\lambda-\lambda_{c l}\right)+\cdots\right] \xi+\left[\varepsilon_{2} B_{2}+\varepsilon_{2} B_{2}^{\prime}\left(\lambda-\lambda_{c l}\right)+\cdots\right] \xi^{2} \\
& +\left[\varepsilon_{3} B_{3}+\varepsilon_{3} B_{3}^{\prime}\left(\lambda-\lambda_{c l}\right)+\cdots\right] \xi^{3}+\left[\varepsilon_{4} B_{4}+\varepsilon_{4} B_{4}^{\prime}\left(\lambda-\lambda_{c l}\right)+\cdots\right] \xi^{4}+\cdots
\end{aligned}
$$

where $A_{1}, A_{1}^{\prime}, A_{1}^{\prime \prime}, \ldots$ and $A_{2}$ vanish because of the condition that the first and second variations of the potential energy are zero in the critical state of equilibrium. In the above $\lambda$ is the applied load parameter, $\lambda_{c l}$ is the classical critical load, $\xi$ is the amplitude of the buckling mode, $A_{2}^{\prime}, A_{2}^{\prime \prime}, A_{3}, A_{3}^{\prime}, \ldots, B_{1}, B_{1}^{\prime}, B_{2}, \ldots$ are constants and $\varepsilon_{1}, \varepsilon_{2}, \varepsilon_{3}$ and $\varepsilon_{4}$ are the amplitudes of various imperfection quantities. That is, these imperfections represent deviations from the idealized model of the structure. Thus, they may appear as initial geometric deformation changes in material specification or any other similar quantity. It should be noted that, for an asymptotic analysis, it is usual to retain only the first non-vanishing term in each of the above square brackets.

In catastrophe theory, the form of a single mode instability is classified according to the first non-vanishing coefficient $A_{n}$, where $n=3,4, \ldots$. The standard form after division by $A_{n}$ and a suitable scaling is

$$
\begin{array}{r}
P E=\xi^{n}+\alpha_{n-2} \xi^{n-2}+\alpha_{n-3} \xi^{n-3}+\cdots+\alpha_{3} \xi^{3}+\alpha_{2} \xi^{2}+\alpha_{1} \xi \\
\text { with } A_{n} \neq 0, \quad A_{n-1}=\cdots A_{2}=0
\end{array}
$$

where $\alpha_{1}, \alpha_{2}, \ldots, \alpha_{n-2}$ are control parameters and are associated with quantities such as applied load(s) and deviations of the real system from the idealized model. Furthermore, all these control parameters are assumed to be zero in the reference state and it is the stability of the perturbations of the reference state which is under consideration.

The fold cuspoid occurs whenever the cubic term of the potential energy is non-zero. The standard form is

$$
P E=\xi^{3}+\alpha_{1} \xi, \quad A_{3} \neq 0 .
$$


Also, the standard form of the cusp model is given by

$$
P E= \pm \xi^{4}+\alpha_{2} \xi^{2}+\alpha_{1} \xi, \quad A_{3}=0, \quad A_{4} \neq 0 .
$$

The swallowtail cuspoid instability occurs when both the cubic and quartic terms of the potential energy vanish (that is, when the quartic term of the cusp model vanishes) and the quintic term is non-zero. Its standard form is

$$
P E=\xi^{5}+\alpha_{3} \xi^{3}+\alpha_{2} \xi^{2}+\alpha_{1} \xi, \quad A_{3}=A_{4}=0, \quad A_{5} \neq 0 .
$$

Continuing, if the quintic term along with the cubic and quartic terms of the potential energy vanish, the next higher form of instability is called the butterfly cuspoid. The standard form of this model is

$$
\begin{aligned}
& P E= \pm \xi^{6}+\alpha_{4} \xi^{4}+\alpha_{3} \xi^{3}+\alpha_{2} \xi^{2}+\alpha_{1} \xi \\
& A_{3}=A_{4}=A_{5}=0, \quad A_{6} \neq 0
\end{aligned}
$$

where the plus or minus sign corresponds respectively to the butterfly catastrophe and to its dual. In the present, because of physical implications, the plus sign corresponds to what is defined as the stable butterfly cuspoid and the negative sign to the unstable butterfly cuspoid. The forms of the instability such as the fold and swallowtail cuspoids which begin with the odd powers of $\xi$ are always unstable at the classical critical load. It is noted that one of the objectives of this paper is to emphasize the importance of the sign of the leading term of the even-ordered catastrophes such as the cusp and the butterfly models. Other higher forms of instability are also of possible interest; however, they are out of the scope of the elementary catastrophes. For example, the standard form of the Wigwam and Star catastrophes are [16], respectively,

$$
\begin{aligned}
& P E=\xi^{7}+\alpha_{5} \xi^{5}+\alpha_{4} \xi^{4}+\alpha_{3} \xi^{3}+\alpha_{2} \xi^{2}+\alpha_{1} \xi \\
& A_{3}=A_{4}=A_{5}=A_{6}=0, \quad A_{7} \neq 0, \\
& P E= \pm \xi^{8}+\alpha_{6} \xi^{6}+\alpha_{5} \xi^{5}+\alpha_{4} \xi^{4}+\alpha_{3} \xi^{3}+\alpha_{2} \xi^{2}+\alpha_{1} \xi \\
& A_{3}=A_{4}=A_{5}=A_{6}=A_{7}=0, \quad A_{8} \neq 0 .
\end{aligned}
$$

Swallowtail cuspoid. By setting the first and second variations of the appropriate potential energy expression to zero, the equilibrium and stability equations of the swallowtail cuspoid are

$$
5 \xi^{4}+3 \alpha_{3} \xi^{2}+2 \alpha_{2} \xi+\alpha_{1}=0, \quad 10 \xi^{3}+3 \alpha_{3} \xi+\alpha_{2}=0 .
$$

The critical load surface is obtained by eliminating the behavior variable from the equilibrium and stability equations. The primary critical load surface is defined to be the first intersection of the equilibrium path with the stability boundary as the applied load is increased from zero. Any subsequent intersection with the stability boundary is termed the secondary surface. Throughout this paper, the primary surfaces are shown as solid lines while the secondary surfaces are shown as dotted lines. All stability boundaries are shown as dash-dot lines. Stable and unstable equilibrium paths are shown as solid and dotted lines respectively.

Fig. 1a shows the swallowtail cuspoid critical load surface of $1-\alpha_{2}$ versus $\alpha_{1}$ for various values of $\alpha_{3}$. The corresponding equilibrium paths for three typical values of $\alpha_{3}$ are shown in Figs. 1b-d. Fig. 1b shows that for a system with positive values of $\alpha_{3}$ and 

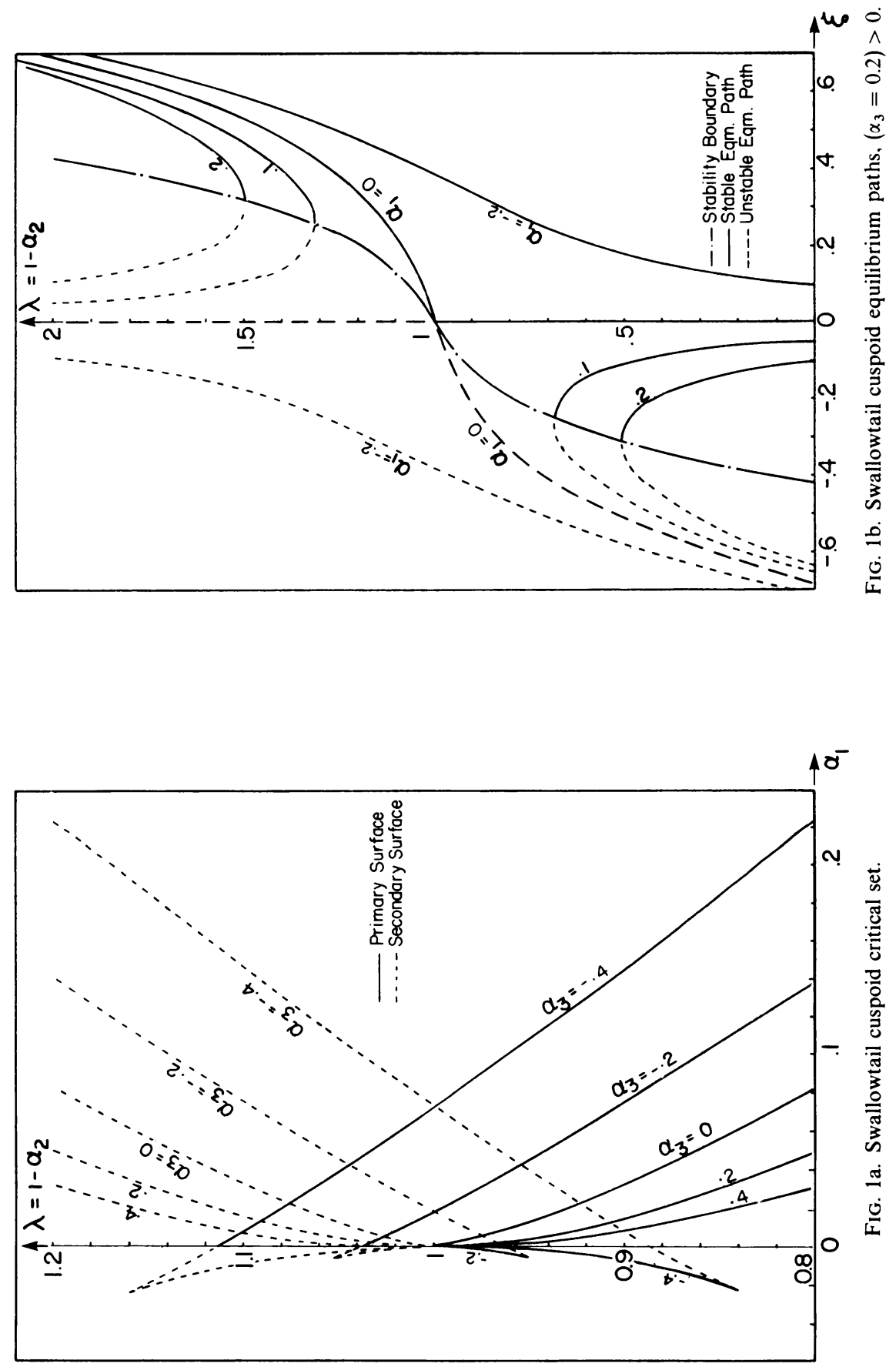

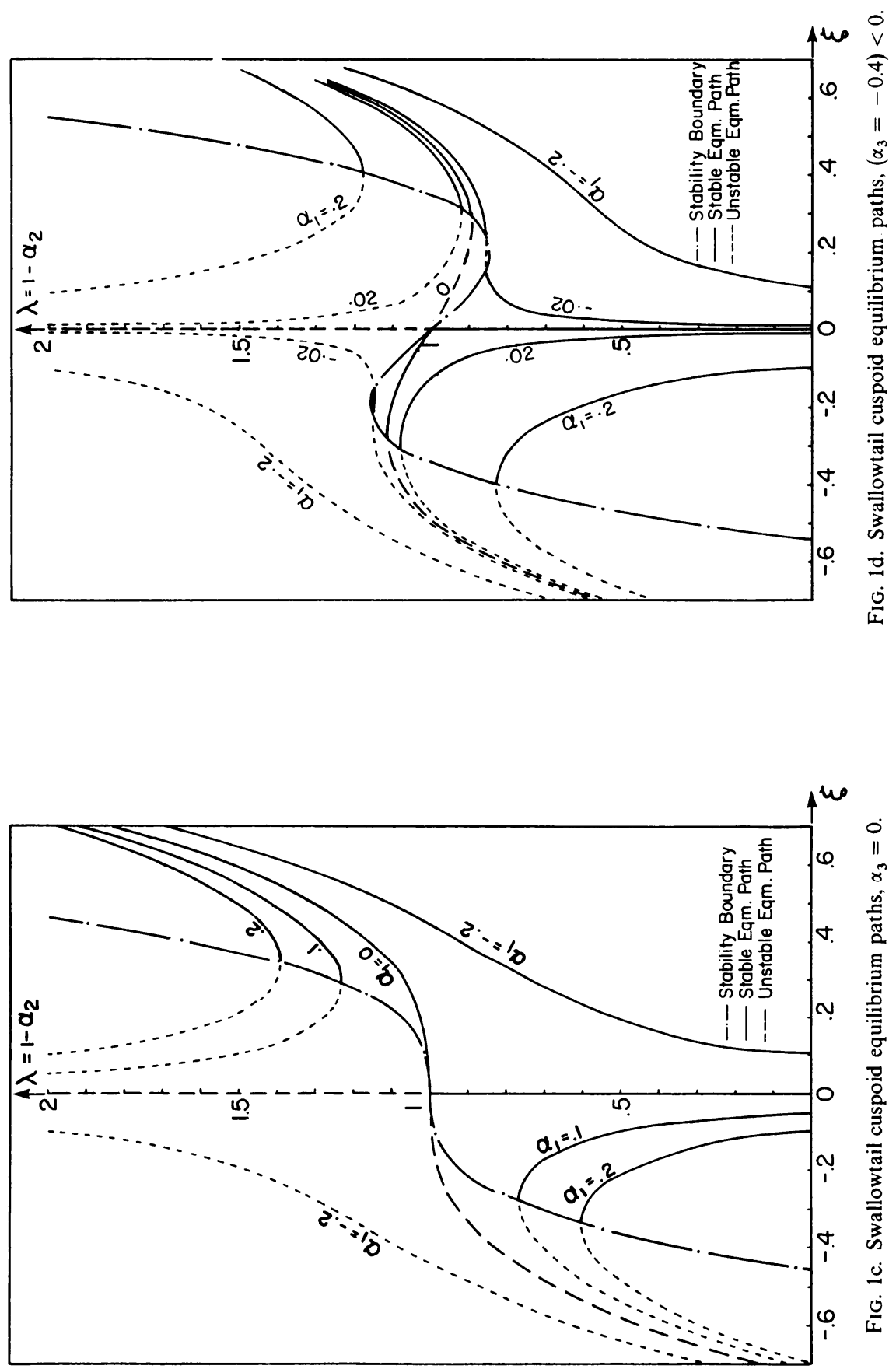
negative values of $\alpha_{1}$, the initially stable equilibrium paths do not intersect the stability boundary. Hence, there is no critical load surface as shown in Fig. 1a. Fig. 1d shows that when $\alpha_{3}$ is negative, the stability boundary has two peaks and therefore, for sufficiently small (in magnitude) negative values of $\alpha_{3}$ the equilibrium paths intersect the stability boundary and the critical surface exists.

Butterfly cuspoid. The equilibrium and stability equations of the stable butterfly cuspoid are, respectively,

$$
6 \xi^{5}+4 \alpha_{4} \xi^{3}+3 \alpha_{3} \xi^{2}+2 \alpha_{2} \xi+\alpha_{1}=0, \quad 15 \xi^{4}+6 \alpha_{4} \xi^{2}+3 \alpha_{3} \xi+\alpha_{2}=0 .
$$

Similarly, for the unstable butterfly cuspoid, these equations become

$$
-6 \xi^{5}+4 \alpha_{4} \xi^{3}+3 \alpha_{3} \xi^{2}+2 \alpha_{2} \xi+\alpha_{1}=0, \quad-15 \xi^{4}+6 \alpha_{4} \xi^{2}+3 \alpha_{3} \xi+\alpha_{2}=0 .
$$

It may be noted that all four of the above equations remain invariant when the parameters $\alpha_{1}, \alpha_{3}$ and deflection $\xi$ change sign simultaneously. Thus the four-dimensional $\left(\alpha_{1}, \alpha_{2}, \alpha_{3}, \alpha_{4}\right)$ critical surface will have reflective symmetry in the sense that when $\alpha_{3}$ is replaced by $-\alpha_{3}$, the surface is symmetric with respect to the $\alpha_{1}$ axis. Further, it is obvious that by changing the signs of $\alpha_{1}, \alpha_{2}, \alpha_{3}$ and $\alpha_{4}$ in the stable butterfly cuspoid, the result is an unstable butterfly cuspoid and vice versa. Similarly, by changing the signs of $\alpha_{2}, \alpha_{4}$ and $\xi$ in the stable butterfly cuspoid, the result is also an unstable butterfly cuspoid and vice versa. Hence, the equilibrium curves and critical surfaces of the unstable butterfly cuspoid are obtained by simply inverting the corresponding graphs of the stable butterfly model (that is, by changing the signs of $\alpha_{1}, \alpha_{2}, \alpha_{3}, \alpha_{4}$ ).

Fig. 2a shows the stable butterfly cuspoid critical load surface when the parameter $\alpha_{4}$ is set to .05. Also, due to symmetry considerations, only negative values of $\alpha_{3}$ are plotted. The critical load surface for positive values of $\alpha_{3}$ can be obtained by reflecting the graph about the load axis. The corresponding equilibrium paths for typical values of $\alpha_{3}$ being -.4 are shown in Fig. 2b. Here, assuming that the applied load is increased from zero, the system will buckle only for sufficiently small negative parameters $\alpha_{1}$. Fig. 2c shows that when $\alpha_{3}=0$ the initially stable system will buckle only when $\alpha_{1}=0$. Again, from symmetry considerations, the equilibrium paths for positive values of $\alpha_{3}$ are similar to Fig. $2 \mathrm{~b}$ except $\alpha_{1}$ and the deflection $\xi$ change sign. This implies that the system will buckle only for sufficiently small positive values of $\alpha_{1}$.

Fig. 3a shows the stable butterfly cuspoid critical load surface for a typical negative value of $\alpha_{4}$ being -.5 . Again, only negative values of $\alpha_{3}$ are plotted due to symmetry. This figure differs from Fig. $2 \mathrm{a}$ in that the primary surfaces exist for sufficiently small positive and negative values of $\alpha_{1}$. This is demonstrated in Figs. 3b,c where the stability boundaries have two minima instead of one. Fig. 3b shows that when $\alpha_{3}=-1$, the relative major peak of the stability boundary corresponds to negative values of $\alpha_{1}$ and thus the primary surface for negative values of $\alpha_{1}$ is higher than the primary surface for positive values of $\alpha_{1}$ as shown in Fig. 3a. The reverse is true for positive values of $\alpha_{3}$ and are therefore not plotted.

Fig. 4a shows the unstable butterfly cuspoid critical load surfaces for a typical negative value of $\alpha_{4}$ being -0.05 . This graph is obtained by inverting Fig. $2 \mathrm{a}$ and then changing the signs of $\alpha_{4}$ and the deflection $\xi$. Although the equilibrium and stability 

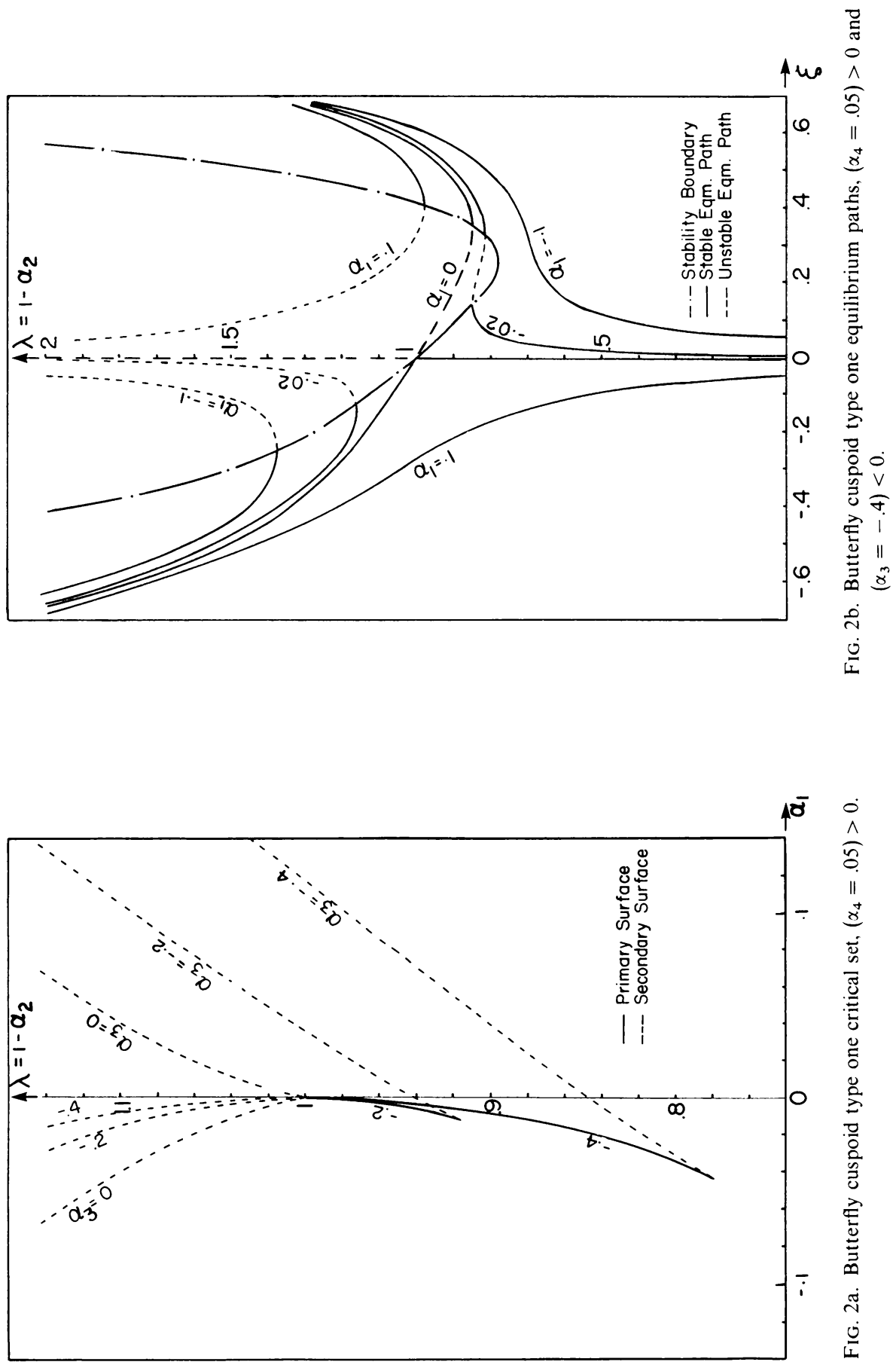

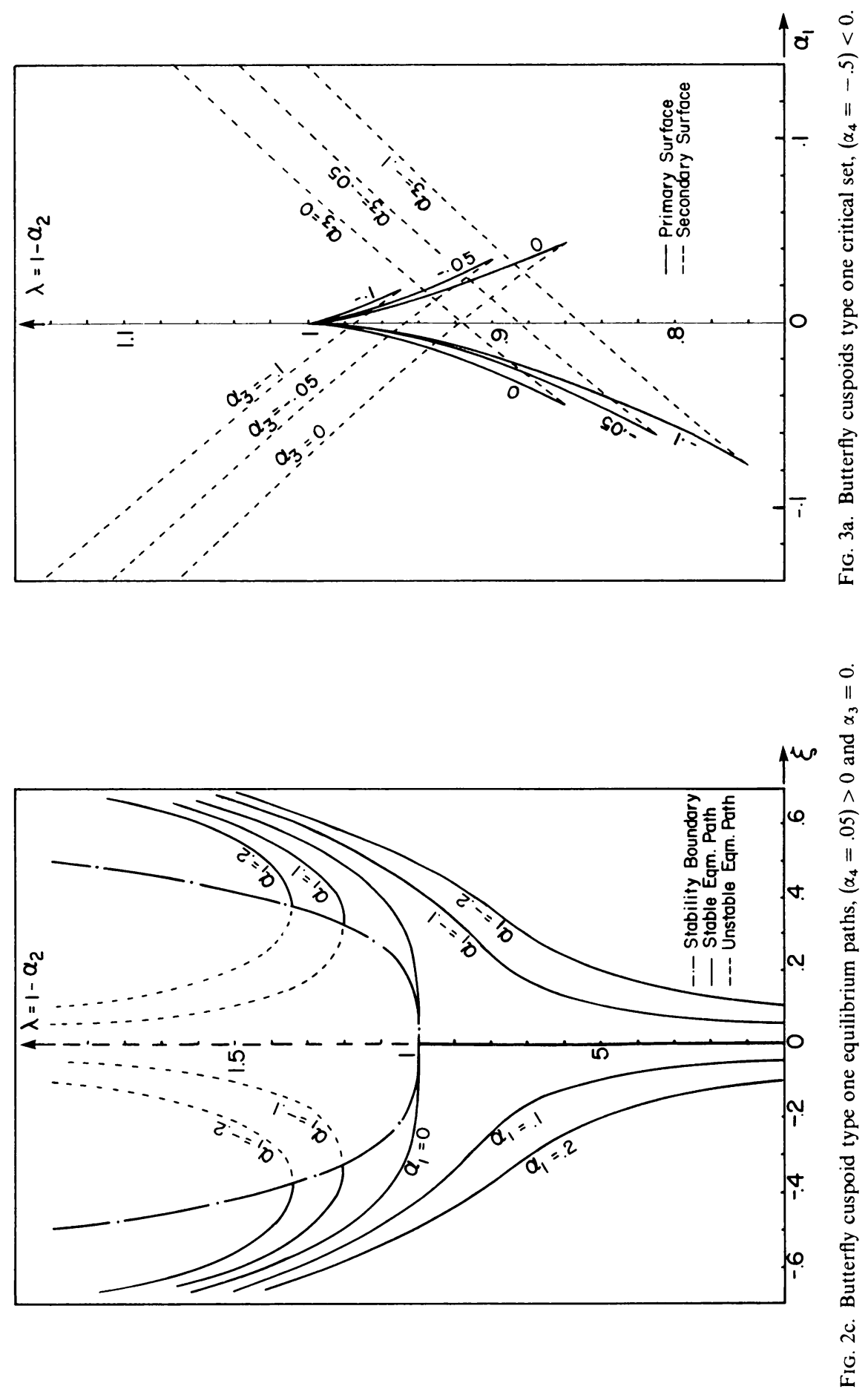

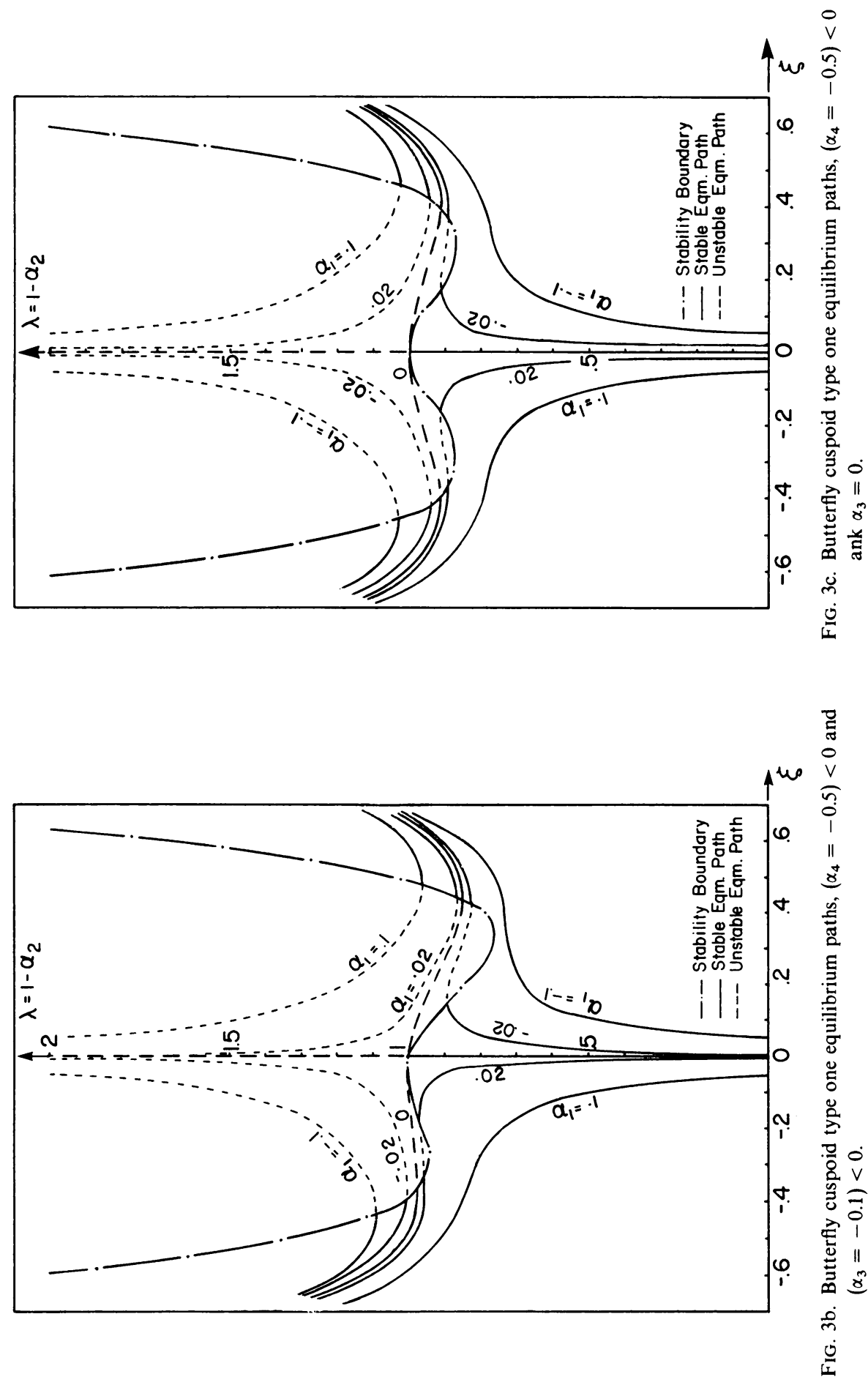


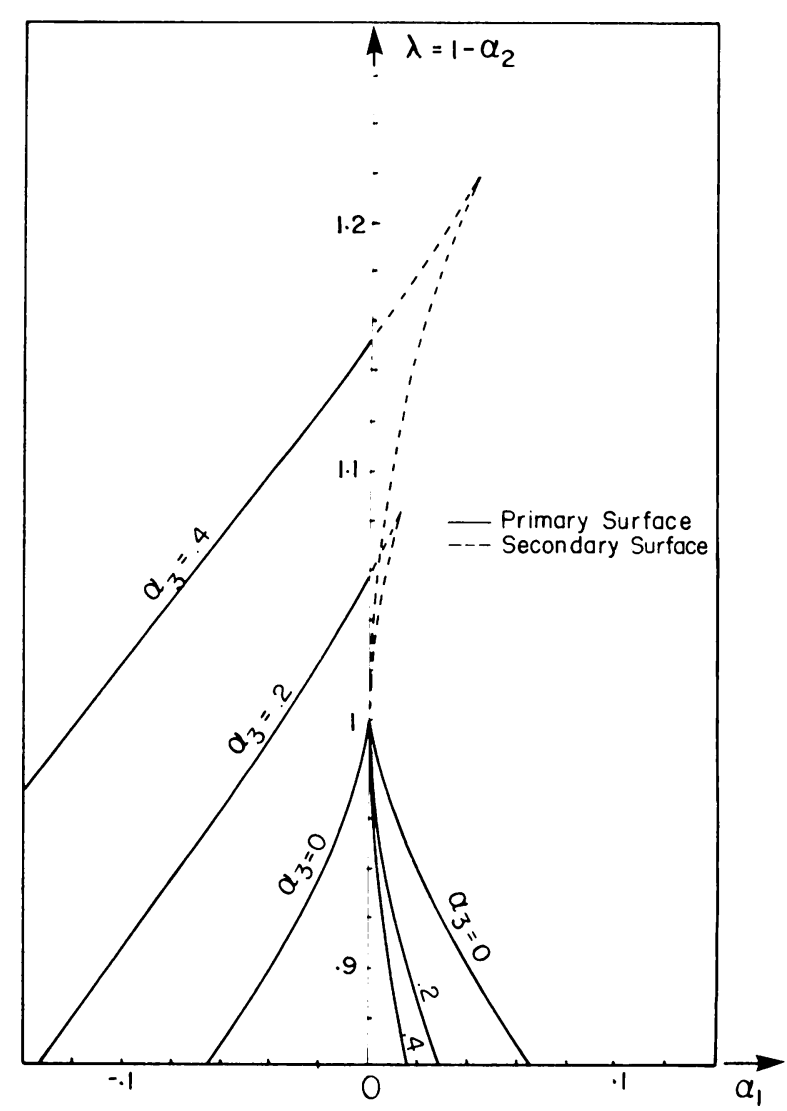

FIG. 4a. Butterfly cuspoid type two critical set, $\left(x_{4}=-.05\right)<0$.

equations of these two figures are the same, the primary surfaces which are of engineering significance are totally different. The system in Fig. 4a is seen to buckle for all values of the imperfection $\alpha_{1}$. This can be explained from the corresponding equilibrium paths of Figs. $4 \mathrm{~b}$ and $4 \mathrm{c}$. Since the stability boundary is inverted to open downwards, there is a severe reduction of the critical load which indicates that the system is more sensitive to imperfections than the stable butterfly cuspoid.

Figs. 5a-c show the unstable butterfly cuspoid critical load surfaces and equilibrium paths when $\alpha_{4}$ is set to 0.5 . Again, this model is shown to be more imperfection-sensitive than the corresponding stable case. Comparison of the equilibrium paths of Fig. $4 \mathrm{a}$ and Fig. $5 \mathrm{~b}$ where the value of $\alpha_{3}$ is fixed at 0.4 shows that an initially stable system with positive values of $\alpha_{1}$ buckled at a relatively higher value of the critical load in Fig. 5b than in Fig. 4b. This is because the stability boundary, Fig. 5b, has two peaks instead of one, thus causing a less severe reduction of the critical load.

Koiter's general theory of initial post-buckling: single-mode systems. In order to demonstrate the present results in an example it is appropriate to first present a summary of the pertinent aspects of Koiter's general theory of elastic buckling and initial postbuckling for single-mode systems. This section does so. The first approximation to the 

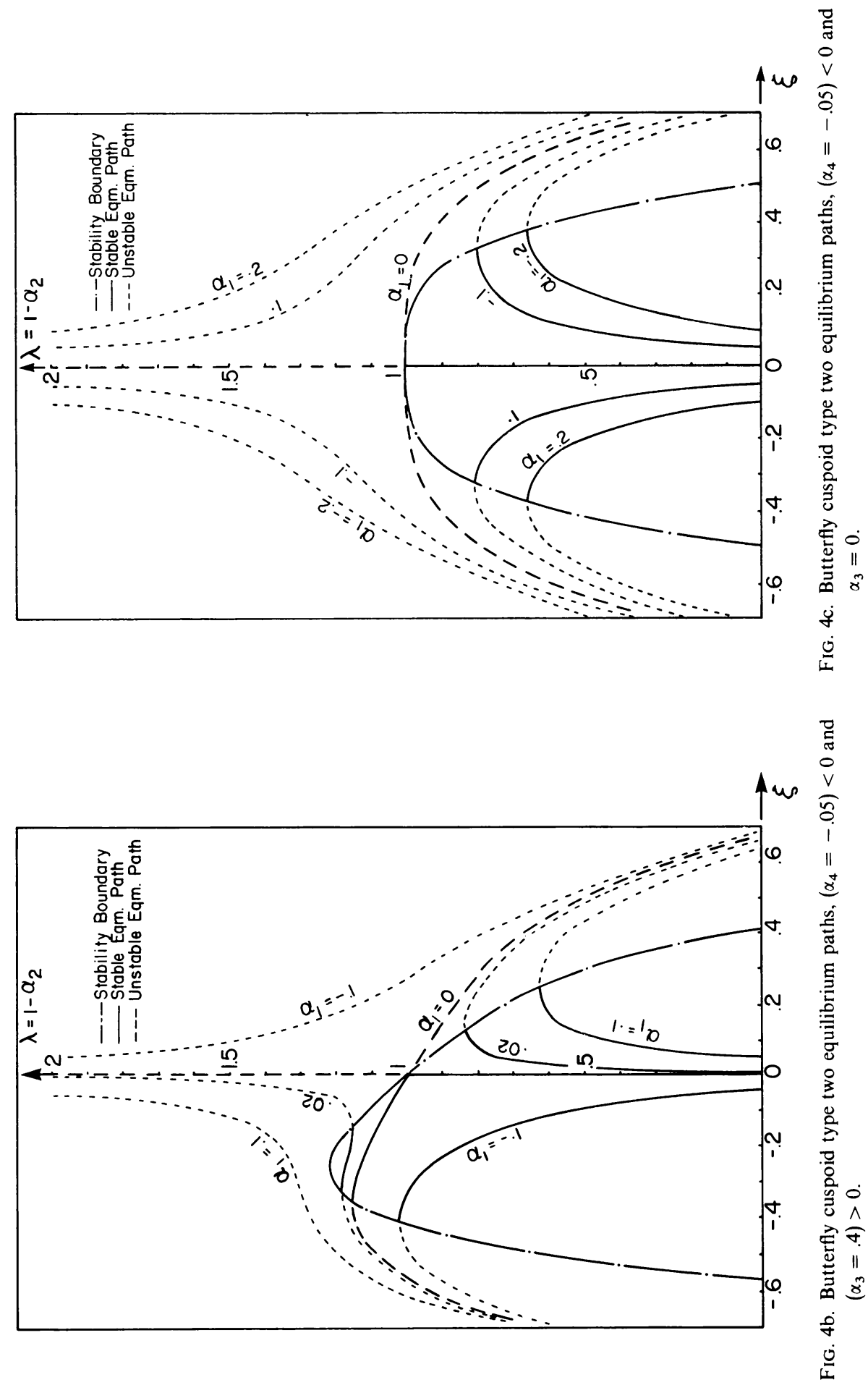

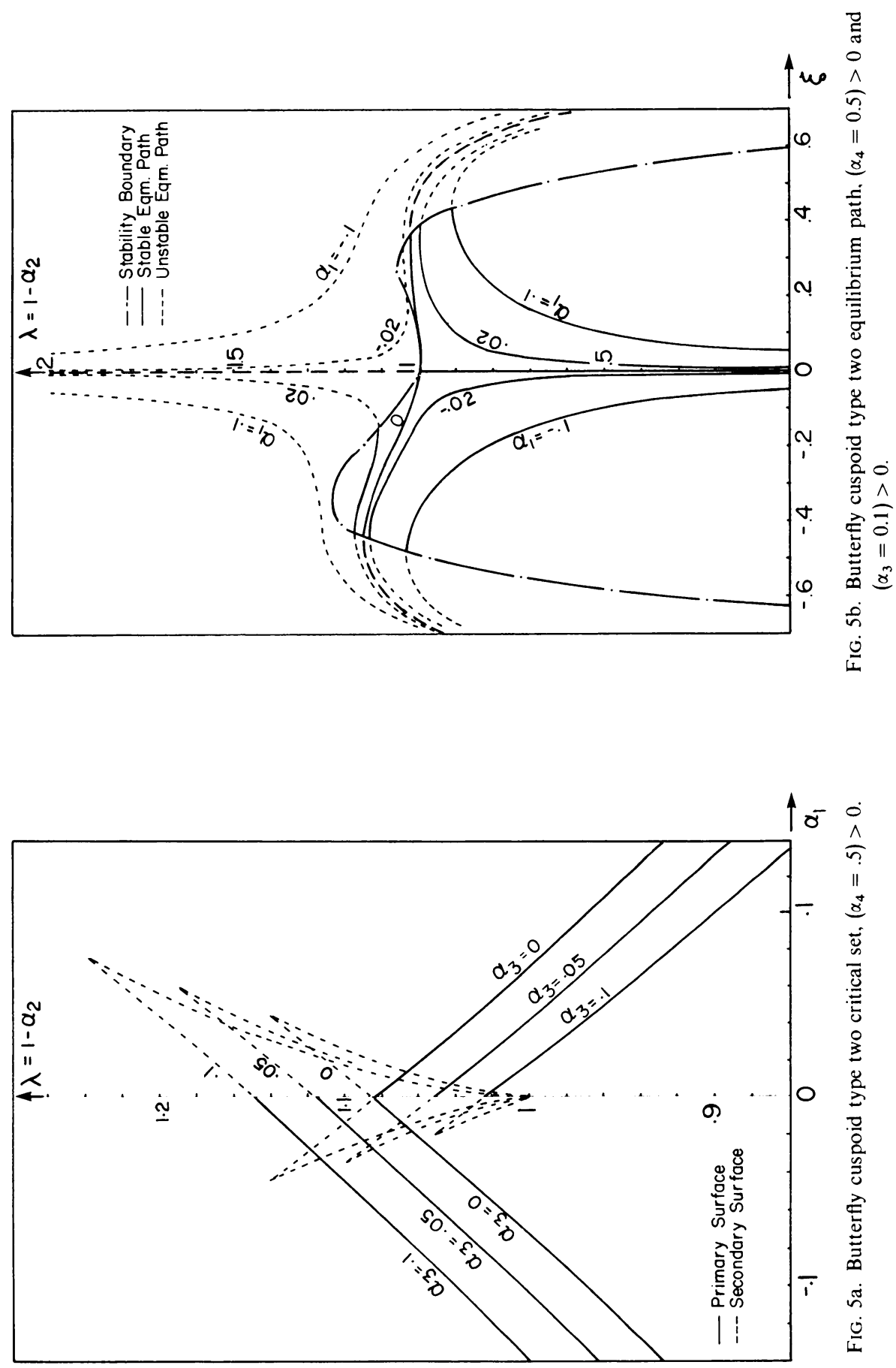


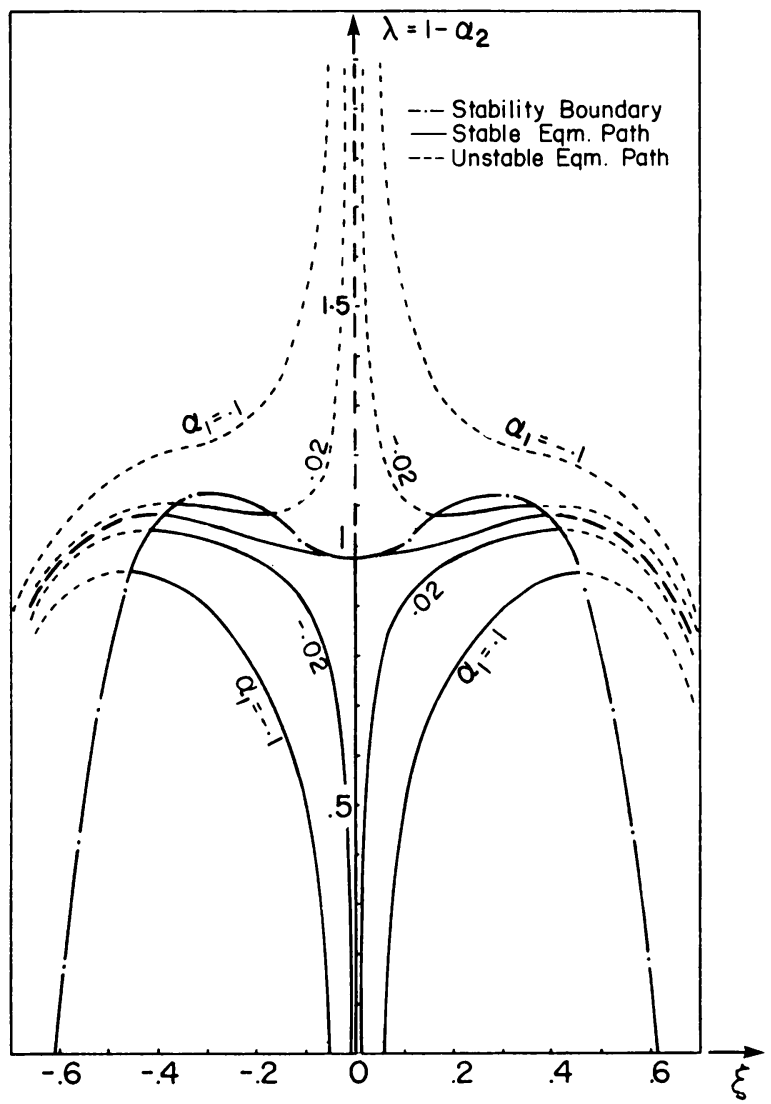

Fig. 5c. Butterfly cuspoid type two equilibrium paths, $\left(\alpha_{4}=.5\right)>0$ and $\alpha_{3}=0$.

potential energy expanded about the classical critical load of the perfect system is

$$
\begin{aligned}
P E=A_{2}^{\prime}\left(\lambda-\lambda_{c l}\right) \xi^{2}+\cdots+A_{n-1}^{\prime}(\lambda & \left.-\lambda_{c l}\right) \xi^{n-1} \\
& +A_{n} \xi^{n}+\cdots+\varepsilon_{1} B_{1} \xi+\cdots+\varepsilon_{n-1} B_{n-1} \xi^{n-1} .
\end{aligned}
$$

Since only the most predominant terms are retained and the prebuckling state is assumed to be linear, Koiter's general theory is valid only for sufficiently small amplitudes of the imperfection parameters. (The swallowtail and butterfly cuspoids correspond to the case when $n=5$ and $n=6$ respectively.) Although many of the terms in the above potential energy expression are considered to be negligibly small in Koiter's approach, they are considered to be essential in order to unfold the particular form of instability properly. The coefficients of the potential energy are

$$
\begin{aligned}
& A_{2}=\frac{d}{d \lambda}\left(P_{2}\left[u_{c r}\right]\right), \\
& A_{3}=\left(P_{3}\left[u_{c r}\right]\right)_{\lambda=\lambda_{c l}}, \quad A_{3}^{\prime}=\left.\frac{d}{d \lambda}\left(P_{3}\left[u_{c r}\right]\right)\right|_{\lambda=\lambda_{c l}}, \\
& A_{4}=\left(P_{4}\left[u_{c r}\right]-P_{2}\left[\phi_{2}\right]\right)_{\lambda=\lambda_{c l}}, \quad A_{4}^{\prime}=\left.\frac{d}{d \lambda}\left(P_{4}\left[u_{c r}\right]-P_{2}\left[\phi_{2}\right]\right)\right|_{\lambda=\lambda_{c l}},
\end{aligned}
$$




$$
\begin{aligned}
& A_{5}=\left(P_{5}\left[u_{c r}\right]+P_{31}\left[u_{c r}, \phi_{2}\right]+P_{12}\left[u_{c r}, \phi_{2}\right]\right)_{\lambda=\lambda_{c l}}, \\
& A_{6}=\left(P_{6}\left[u_{c r}\right]+P_{41}\left[u_{c r}, \phi_{2}\right]+P_{22}\left[u_{c r}, \phi_{2}\right]+P_{3}\left[\phi_{2}\right]-P_{2}\left[\psi_{3}\right]\right)_{i=\lambda_{c l}} \text {, } \\
& B_{1}=\left(Q_{1}\left[u_{c r}\right]\right)_{\lambda=\lambda_{c l}}, \quad B_{2}=\left(Q_{2}\left[u_{c r}\right]-P_{11}\left[\bar{\phi}_{0}, \phi_{2}\right]\right)_{\lambda=\lambda_{c l}} \text {, } \\
& B_{3}=\left(Q_{3}\left[u_{c r}\right]-P_{11}\left[\bar{\phi}_{0}, \phi_{3}\right]-P_{11}\left[\bar{\phi}_{1}, \phi_{2}\right]\right)_{i=i_{c l}} \text {, } \\
& B_{4}=\left(Q_{4}\left[u_{c r}\right]-P_{11}\left[\bar{\phi}_{1}, \phi_{3}\right]-P_{11}\left[\bar{\phi}_{2}, \phi_{2}\right]\right)_{\lambda=\lambda_{c l}} \text {. }
\end{aligned}
$$

In the above $P_{m n}[u, v]$ represents terms in the potential energy of the perfect system which are independent of the imperfection and are of $m$ th order in the deflection $u$ and $n$th order in $v$, and $Q_{n}[u]$ represents terms in the potential energy which are linear both in the applied load and generalized imperfection and are of $n$th order in the deflection $u$. The functions $\phi_{2}, \phi_{3}, \psi_{3}$ of the perfect system and $\bar{\phi}_{0}, \bar{\phi}_{1}$, and $\bar{\phi}_{2}$ of the perfect system are obtained by solving the appropriate differential equations, that is, from [2],

$$
\begin{aligned}
& {\left[P_{11}\left[\phi_{2}, \delta u\right]+P_{21}\left[u_{c r}, \delta u\right]-\left(\frac{3 P_{3}\left[u_{c r}\right]}{2 T_{2}\left[u_{c r}\right]}\right) T_{11}\left[u_{c r}, \delta u\right]\right]_{i=\lambda_{c l}}=0,} \\
& {\left[P_{11}\left[\phi_{3}, \delta u\right]+P_{31}\left[u_{c r}, \delta u\right]-\left(\frac{4 P_{4}\left[u_{c r}\right]}{2 T_{2}\left[u_{c r}\right]}\right) T_{11}\left[u_{c r}, \delta u\right]\right]_{i=i_{c l}}=0,} \\
& {\left[P_{11}\left[\psi_{3}, \delta u\right]+P_{31}\left[u_{c r}, \delta u\right]+P_{111}\left[u_{c r}, \phi_{2}, \delta u\right]\right.} \\
& \left.-\frac{4 P_{4}\left[u_{c r}\right]+2 P_{21}\left[u_{c r}, \phi_{2}\right]}{2 T_{2}\left[\phi_{1}\right]} T_{11}\left[\psi_{1}, \delta u\right]\right]_{i=\lambda_{c l}}=0, \\
& {\left[P_{11}\left[\phi_{0}, \delta u\right]+Q_{1}[\delta u]-\left(\frac{Q_{1}\left[u_{c r}\right]}{2 T_{2}\left[u_{c r}\right]}\right) T_{11}\left[u_{c r}, \delta u\right]\right]_{i=\lambda_{c l}}=0,} \\
& {\left[P_{11}\left[\bar{\phi}_{1}, \delta u\right]+Q_{11}\left[u_{c r}, \delta u\right]-\left(\frac{Q_{2}\left[u_{c r}\right]}{T_{2}\left[u_{c r}\right]}\right) T_{11}\left[u_{c r}, \delta u\right]\right]_{\lambda=\lambda_{c l}}=0,} \\
& {\left[P_{11}\left[\phi_{2}, \delta u\right]+Q_{21}\left[u_{c r}, \delta u\right]-\left(\frac{3 Q_{3}\left[u_{c r}\right]}{2 T_{2}\left[u_{c r}\right]}\right) T_{11}\left[u_{c r}, \delta u\right]\right]_{i=i_{c l}}=0,}
\end{aligned}
$$

where $\delta u$ is a variational quantity and the functions $\phi_{2}, \phi_{3}, \psi_{3}, \bar{\phi}_{0}, \bar{\phi}_{1}$ and $\bar{\phi}_{2}$ satisfy the orthogonality condition

$$
\begin{aligned}
T_{11}\left[u_{c r}, \phi_{2}\right] & =T_{11}\left[u_{c r}, \phi_{3}\right]=T_{11}\left[u_{c r}, \psi_{3}\right]=T_{11}\left[u_{c r}, \bar{\phi}_{0}\right] \\
& =T_{11}\left[u_{c r}, \bar{\phi}_{1}\right]=T_{11}\left[u_{c r}, \bar{\phi}_{2}\right]=0
\end{aligned}
$$

and $T_{11}[u, v]$ can be taken as the inner product of the two vector quantities $u$ and $v$.

Example: beam on a nonlinear elastic foundation. The single-mode buckling problems discussed will be considered within the context of the stability analysis of a beam resting on a nonlinear elastic foundation subjected to a concentrated axial compressive load $P$ (positive for compression). The potential energy of the system consists of the sum of the strain energy of the beam due to bending and the strain energy absorbed by the 
elastic foundation and the work term, that is,

$$
\begin{aligned}
P E= & U_{0}+U_{F}-\text { Work } \\
= & \frac{1}{2} E I \int_{0}^{L}\left\{\frac{W_{, X X}^{2}}{1-W_{, X}^{2}}\right\} d X \\
& +\int_{0}^{L} \int_{0}^{W}\left\{K_{1} W+K_{2} W^{2}+K_{3} W^{3}+\cdots\right\} d W d X-P \int_{0}^{L}\left\{1-\sqrt{1-W_{, X}^{2}}\right\} d X .
\end{aligned}
$$

In the above, $E$ is Young's modulus, $I$ is the moment of inertia of the beam, $W$ is the vertical deflection, $X$ is the horizontal coordinate and $L$ is the length of the beam and $K_{1}$, $K_{2}, K_{3}, \ldots$ are the spring constants of the elastic foundation. Introducing the following nondimensional quantities $x, w, \lambda, k_{1}, k_{2}, k_{3}, \ldots$ defined by

$$
x=X / L, \quad w=W / L, \quad \lambda=P L^{2} / E I, \quad k_{i}=K_{i} L^{i+3} / E I, \quad i=1,2,3, \ldots,
$$

the potential energy expression becomes

$$
\begin{aligned}
P E= & \left(\frac{E I}{2 L}\right) \int_{0}^{1}\left[\frac{w_{, x x}^{2}}{1-w_{, x}^{2}}\right] d x \\
& \left.+2 \int_{0}^{1}\left[\frac{1}{2} k_{1} w^{2}+\frac{1}{3} k_{2} w^{3}+\frac{1}{4} k_{3} w^{4}+\cdots\right] d x+2 \lambda \int_{0}^{1}\left[-1+\sqrt{1-w_{, x}^{2}}\right] d x\right\} .
\end{aligned}
$$

By grouping the terms according to the order of the deflection, the potential energy can be expressed as

$$
P E=P_{2}^{\lambda}[u]+P_{3}^{\lambda}[u]+P_{4}^{\lambda}[u]+P_{5}^{\lambda}[u]+P_{6}^{\lambda}[u]+\cdots
$$

where

$$
\begin{aligned}
& P_{2}^{\lambda}[u]=\frac{E I}{2 L} \int_{x=0}^{1}\left(w_{, x x}^{2}+k_{1} w^{2}\right)-\lambda w_{, x}^{2} d x \\
& P_{3}^{\lambda}[u]=\left(\frac{E I}{2 L}\right)\left(\frac{2}{3} k_{2} \int_{x=0}^{1} w^{3} d x\right) \\
& P_{4}^{\lambda}[u]=\left(\frac{E I}{2 L}\right) \int_{x=0}^{1}\left(w_{, x x}^{2} w_{, x}^{2}+\frac{1}{2} k_{3} w^{4}\right)-\frac{1}{4} \lambda w_{, x}^{4} d x \\
& P_{5}^{\lambda}[u]=\left(\frac{E I}{2 L}\right)\left(\frac{2}{5} k_{4} \int_{x=0}^{1} w^{5} d x\right) \\
& P_{6}^{\lambda}[u]=\left(\frac{E I}{2 L}\right) \int_{x=0}^{1}\left(w_{, x x}^{2} w_{, x}^{4}+\frac{1}{3} k_{5} w^{6}\right)-\frac{1}{8} \lambda w_{, x}^{6} d x .
\end{aligned}
$$

It is worth noting that the odd functionals $P_{3}^{\lambda}[u], P_{5}^{\lambda}[u], P_{7}^{\lambda}[u], \ldots$ are independent of the applied load and that $\lambda$ is a positive quantity which is assumed to increase from zero. Furthermore, the prebuckling state admits the zero-displacement solution.

Upon setting the first variation of the quadratic terms $P_{2}^{\lambda}[u]$ to zero, the differential equation for classical buckling is

$$
w_{, x \boldsymbol{x x x}}+\lambda w_{, x x}+k_{1} w=0
$$


where the forced boundary conditions are $w=0$ at $x=0,1$, and the natural boundary conditions are $w_{, x x}=0$ at $x=0,1$. The solution to this eigenvalue problem is

$$
w=\xi_{m} \sum_{n=1}^{\infty} \sin m \pi x
$$

so that the eigenvalues are

$$
\lambda_{m}=\frac{m^{4} \pi^{4}+k_{1}}{m^{2} \pi^{2}} \quad m=1,2, \ldots
$$

where $m$ is a positive integer. By minimizing $\lambda_{m}$ with respect to $m$, it follows that the critical wave number $m_{c r}=j$ must lie somewhere near the value $\left(4 \sqrt{ } k_{1}\right) / \pi$. In addition, for

$$
\lambda_{c l}=\lambda_{j}=\frac{j^{4} \pi^{4}+k_{1}}{j^{2} \pi^{2}},
$$

$\lambda_{l}>\lambda_{j}, l<j$ and $\lambda_{n}>\lambda_{j}, n>j$. Further, the least upper bounds may be shown to occur for $l=j-1$ and $n=j+1$ respectively. Thus it follows that the critical wave number $j$ is obtained from the inequalities

$$
(j-1)^{2} j^{2} \pi^{4}<k_{1}<(j+1)^{2} j^{2} \pi^{4} .
$$

In order to investigate the stability of the critical state, it is necessary to consider higher-order terms in the potential energy. Thus, substituting $w=w_{c r}$ into $P_{3}^{\lambda}[u]$, the quantity $A_{3}$ is found to be $\left(m_{c r}=j\right)$

$$
\begin{aligned}
A_{3} \xi^{3}=P_{3}^{\lambda}\left[u_{c r}\right] & =\left(\frac{E I}{2 L} \xi^{3}\right)\left(\frac{2}{3} k_{2} \int_{x=0}^{1}(\sin j \pi x)^{3} d x\right)=0, \quad j=\text { even } \\
& =\left(\frac{E I}{2 L} \xi^{3}\right)\left(\frac{8}{9 j \pi} k_{2}\right), \quad j=\text { odd. }
\end{aligned}
$$

Thus, the swallowtail or butterfly catastrophes will only occur if $k_{2}=0$, or if the critical wave number $j$ is even.

In order to compute $A_{4}$, it is necessary to solve the differential equation for $\phi_{2}$, that is,

$$
\delta P_{2}\left[\phi_{2}\right]=-\delta P_{3}\left[u_{c r}\right]
$$

If it is assumed that $k_{2}$ is zero, the right-hand side of the above equation is zero. Furthermore, since the displacement $\phi_{2}$ must be orthogonal to the buckling mode $w_{c r}$, it follows that $\phi_{2}=0$. Thus the quantity $A_{4}$ becomes

$$
\begin{aligned}
A_{4} \xi^{4} & =\left.P_{4}\left[u_{c r}\right]\right|_{i=\lambda_{c l}}=\left.\left(\frac{E I}{2 L}\right)\right|_{0} ^{1} w_{c r, x x}^{2} w_{c r, x}^{2}+\frac{1}{2} k_{3} w_{c r}^{4}-\frac{1}{4} \lambda_{c l} w_{c r, x}^{4} d x \\
& =\left(\frac{E I}{2 L} \xi^{4}\right)\left[\frac{1}{32} j^{6} \pi^{6}-\frac{3}{32} j^{2} \pi^{2} k_{1}+\frac{3}{16} k_{3}\right] .
\end{aligned}
$$

It follows from the expression for $A_{4}$ that the only situation in which a higher-order catastrophe will arise is when $A_{4}=0$, or

$$
\frac{1}{3}(j \pi)^{6}+2 k_{3}=(j \pi)^{2} k_{1} .
$$


Using our previous result that $\phi_{2}=0$, the quantity $A_{5}$ is found to be

$$
\begin{aligned}
A_{5} \xi^{5}=\left.P_{5}\left[u_{c r}\right]\right|_{i=\lambda_{c l}} & =\left(\frac{E I}{2 L}\right)\left(\frac{2}{5} k_{4} \int_{x=0}^{1} w_{c r}^{5} d x\right)=0, \quad j=\text { even } \\
& =\left(\frac{E I}{2 L} \xi^{5}\right)\left(\frac{32}{75 j \pi} k_{4}\right), \quad j=\text { odd. }
\end{aligned}
$$

Thus with $k_{2}=0$ and by relating $k_{1}$ and $k_{3}$, one obtains $A_{3}=A_{4}=0$. The swallowtail catastrophe occurs when $k_{4}$ is non-zero and $j$ is an odd integer.

The butterfly catastrophe arises if $A_{5}=0$, which implies that the spring constant $k_{4}$ is zero or that $j$ is odd. The quantity $A_{6}$ is, using our previous result that $\phi_{2}=0$,

$$
A_{6} \xi^{6}=\left.P_{6}\left[u_{c r}\right]\right|_{\lambda=\lambda_{c l}}-\left.P_{2}\left[\psi_{3}\right]\right|_{\lambda=\lambda_{c l}}
$$

where the differential equation for the function $\psi_{3}$ is

$$
\left.\delta P_{2}\left[\psi_{3}\right]\right|_{\lambda=\lambda_{c l}}=-\left.\delta P_{4}\left[u_{c r}\right]\right|_{\lambda=i_{c l}} .
$$

This equation, when expanded, becomes

$$
\begin{aligned}
2\left(\psi_{3, x x x x}+k_{1} \psi_{3}+\right. & \left.\lambda_{c l} \psi_{3, x x}\right)\left(\delta \psi_{3}\right) \\
& =\left[2\left(w_{c r, x x}^{2} w_{c r . x}\right)_{, x}-2\left(w_{c r, x}^{2} w_{c r, x x}\right)_{, x x}-2 k_{3} w_{c r}^{3}-3 \lambda_{c l} w_{c r, x}^{2} w_{c r, x x}\right](\delta w)
\end{aligned}
$$

with the forced and natural boundary conditions being those of the buckling mode. Substituting the buckling mode $w_{c r}$ into the right-hand side of the above equation and assuming the solution for $\psi_{3}$ in the form

$$
\psi_{3}=\sum_{n=1}^{\infty} c_{n} \sin n \pi x,
$$

then the result is obtained that

$$
c_{3}=\frac{\xi^{3}(j \pi)^{2}\left[3 k_{1}-7(j \pi)^{4}\right]}{48\left[9(j \pi)^{4}-k_{1}\right]}
$$

and

$$
c_{1}=c_{2}=c_{4}=c_{5}=\cdots=0 .
$$

Substituting $u=\psi_{3}$ into $P_{2}[u]$ and carrying out the integration yields

$$
\left.P_{2}\left[\psi_{3}\right]\right|_{\lambda=\lambda_{c l}}=\frac{E I}{2 L}\left(c_{3}\right)^{2}\left(36 j^{4} \pi^{4}-4 k_{1}\right) .
$$

Thus, the term $A_{6} \xi^{6}$ is found to be

$$
\begin{aligned}
A_{6} \xi^{6} & =\left.P_{6}\left[u_{c r}\right]\right|_{\lambda=\lambda_{c l}}-\left.P_{2}\left[\psi_{3}\right]\right|_{i=\lambda_{c l}} \\
& =\left(\xi^{6} \frac{E I}{2 L}\right)\left\{\left(\frac{1}{16}\right)\left(\frac{3}{8} j^{8} \pi^{8}-\frac{5}{8} j^{4} \pi^{4} k_{1}+\frac{5}{3} k_{5}\right)-\left(c_{3}\right)^{2}\left(36 j^{4} \pi^{4}-4 k_{1}\right)\right\},
\end{aligned}
$$

where it should be noted that the sign of $A_{6}$ dictates whether the problem is classified as stable or unstable butterfly catastrophe.

In order to unfold a catastrophe where the leading term in the potential energy is of the form $A_{n} \xi^{n}$, it is necessary to include all those terms which involve $\xi, \ldots, \xi^{n-2}$ and to 
perturb the coefficients of these terms about zero. Thus, in the context of the present example, the linear term in $\xi$, due to the influence of a geometric imperfection in the form of the buckling mode, is

$$
\frac{1}{\lambda_{c l}} P_{11}^{\prime}\left[u_{c r}, \bar{u}\right]=\frac{E I}{2 L}\left(\frac{-\lambda}{\lambda_{c l}}\right)(\xi \bar{\xi}) j^{2} \pi^{2},
$$

while the $\xi^{2}$ term is

$$
\left(\frac{\lambda-\lambda_{c l}}{\lambda_{c l}}\right) \frac{\partial}{\partial \lambda}\left(P_{2}^{\prime}\left[u_{c r}\right]\right)=\frac{E I}{2 L}\left(1-\frac{\lambda}{\lambda_{c l}}\right)\left(\frac{1}{2} \xi^{2} j^{2} \pi^{2}\right) .
$$

Moreover, the remaining cubic and quartic unfolding terms are obtained by replacing $k_{1}$, $k_{2}, k_{3}$ and $k_{4}$ by $k_{1}+\varepsilon_{k_{1}}, k_{2}+\varepsilon_{k_{2}}, k_{3}+\varepsilon_{k_{3}}$ and $k_{4}+\varepsilon_{k_{4}}$ respectively, where $\varepsilon_{k_{1}}, \varepsilon_{k_{2}}, \varepsilon_{k_{3}}$ and $\varepsilon_{k_{4}}$ are small perturbations of the spring constants. Doing so yields

$$
\begin{aligned}
A_{3}^{\prime} \xi^{3} & =\frac{E I}{2 L} \xi^{3} \frac{8}{9 j \pi} \varepsilon_{k_{2}}+B_{3}, \quad j=\text { odd }, \\
& =B_{3}, \quad j=\text { even, } \\
A_{4}^{\prime} \xi^{4} & =\left(\frac{E I}{2 L} \xi^{4}\right)\left[\frac{1}{32} j^{6} \pi^{6}-\frac{3}{32} j^{2} \pi^{2} \varepsilon_{k_{1}}+\frac{3}{16} \varepsilon_{k_{3}}\right]+B_{4},
\end{aligned}
$$

and

$$
\begin{aligned}
A_{5}^{\prime} \xi^{5} & =\left(\frac{E I}{2 L} \xi^{5}\right)\left(\frac{32}{75 j \pi} \varepsilon_{k_{4}}\right)+B_{5}, \quad j=\text { odd }, \\
& =B_{5}, \quad j=\text { even. }
\end{aligned}
$$

In the above expressions, $B_{3}$ and $B_{5}$ are obtained by solving the appropriate differential equations (as shown in Eqs. (13)). These terms represent the influence of initial geometric imperfection on the cubic and quartic terms respectively.

It should be noted that $\varepsilon_{k_{1}}, \varepsilon_{k_{2}}, \varepsilon_{k_{3}}$ and $\varepsilon_{k_{4}}$ and $\lambda-\lambda_{c l}$ as well as the amplitude of the geometric imperfection constitute six independent control parameters. It is the sum of their influence, rather than the individual perturbation, which is important. For example, the individual influence of $\varepsilon_{k_{1}}$ and $\varepsilon_{k_{3}}$ is of little interest and it is the aggregate sum of these two perturbations which form the $A_{4}^{\prime}$ coefficient which is of significance. Furthermore, the term $\xi^{n-1}$ (i.e., $A_{5}^{\prime}$ in the case of butterfly catastrophe and $A_{4}^{\prime}$ in the case of swallowtail catastrophe) can be made to vanish by a straightforward coordinate transformation.

Finally, inspection of the expanded potential energy expression shows that the singlemode system is classified as the swallowtail catastrophe when $n=5$ and as the butterfly catastrophe (stable or unstable depending on the sign of $A_{6}$ ) when $n=6$. Consequently, the equilibrium paths and critical surfaces plotted provide useful information on the buckling and initial post-buckling analysis of a beam on a nonlinear elastic foundation.

Concluding remarks. Since the swallowtail and butterfly models in catastrophe theory are the only ones among the elementary catastrophes which have not been solved, the present paper completes the analysis of the seven elementary catastrophes from the point of view of the theory of elastic stability. 
The three-dimensional critical load surface of the swallowtail cuspoid has been analyzed in its entirety. It corresponds to a stability problem where the first nonvanishing term of the potential energy at the singularity is quintic; thus, the structural configuration at the classical critical load is unstable. The four-dimensional butterfly cuspoid critical load surface has been analyzed by choosing typical values of one of the four control parameters. It arises in a stability problem where the first non-vanishing term of the potential energy at the singularity is $\xi^{6}$. The positive sign corresponds to the stable butterfly cuspoid and the negative sign corresponds to the unstable butterfly cuspoid. The latter is found to be more imperfection-sensitive because of the stability boundary opens downward rather than upward.

The equilibrium paths of the swallowtail model are antisymmetric with respect to the load axis. On the other hand, the equilibrium paths of the stable and unstable butterfly models are symmetric with respect to the load axis. Unlike the fold model, the slope of the load-deflection graph of the cusp, swallowtail, butterfly and any higher order cuspoids is always zero. Hence, the horizontal line $\lambda=\lambda_{c l}$ represents the first approximation of the post-buckling deflection path which is valid for vanishingly small control parameters.

Koiter's theory for single-mode structural system has been extended in a somewhat modified form. This is because catastrophe theory requires that in order to unfold the model, or present the form of the instability in its most general form, it is necessary to include the imperfection terms involving $B_{2}, B_{3}, \ldots, B_{n-2}$ in addition to the $B_{1}$ term which involves the initial geometric deviation of the system. Practical application of the swallowtail and butterfly in single-mode structural system is shown in an axially loaded beam on a nonlinear elastic foundation. Finally, it should be emphasized that the results presented in this paper are asymptotic in a sense that they are valid for sufficiently small regions near the critical point.

\section{REFERENCES}

[1] R. Thom, Structural stability and morphogenesis, W. A. Benjamin Inc. (1975)

[2] W. T. Koiter, On the stability of an elastic equilibrium, doctoral thesis, Delft, The Netherlands, 1945 (NASA Tech. Trans. F10,833,1967 and AFFDL-TR-70-25, 1970)

[3] J. M. T. Thompson and G. W. Hunt, A general theory of elastic stability, John Wiley and Sons, 1973

[4] B. Budiansky and J. W. Hutchinson, Dynamic buckling of imperfection-sensitive structures, in Proc. 11th Int. Cong. of App. Mech., Munich, 1964, edited by H. Gortler, Springer-Verlag (1966), pp. 636-651

[5] J. M. T. Thompson and G. W. Hunt, Towards a unified bifurcation theory, J. Appl. Math. Phys. 26, 581-603 (1975)

[6] D. Hui, The parabolic umbilic catastrophe and its application in the theory of elastic stability, M.A. Sc. thesis, Univ. of Toronto, 1977

[7] D. Hui and J. S. Hansen, Buckling and initial post-buckling of a two-mode infinite plate on a non-linear elastic foundation: an application of catastrophe theory, to be published

[8] V. Tvergaard, Imperfection-sensitivity of a wide integrally stiffened panel under compression, Int. J. Solids Struct. 9, 177-192 (1973)

[9] W. T. Koiter, Buckling and post-buckling behavior of a cylindrical panel under axial compression, Trans. Nat. Aero. Res. Inst., Amsterdam, NLL-TR-S476 (1956)

[10] W. Stephens, Imperfection sensitivity of axially compressed stringer reinforced cylindrical panels under internal pressure, AIAA J. 9, 1713-1719 (1971)

[11] J. W. Hutchinson and J. C. Amazigo, Imperfection-sensitivity of eccentrically stiffened cylindrical shells, AIAA J. 5, 392-401 (1967)

[12] J. W. Hutchinson, Buckling and initial post-buckling behavior of oval cylindrical shells under axial compression, J. App. Mech. 35, 66-72 (1968) 
[13] N. R. Bauld, Jr., Imperfection sensitivity of axially compressed stringer reinforced cylindrical sandwich panels, Int. J. Solids Struct. 10, 833-902 (1974)

[14] J. R. Fitch, The buckling and post-buckling behavior of spherical caps under concentrated load, Int. J. Solids Struct. 4, 421-446 (1968)

[15] J. R. Fitch and B. Budiansky, The buckling and post-buckling of spherical caps under axisymmetric load, AIAA J. 8, 686-692 (1970)

[16] A. E. R. Woodcock and T. Poston, A geometric study of the elementary catastrophes, Springer-Verlag (1974)

[17] E. C. Zeeman, Catastrophe theory, selected papers 1972-1977, Addison-Wesley Publishing Co. Inc. (1977)

[18] T. Poston and I. N. Stewart, Taylor expansions and catastrophes, Pitman Publishing Ltd. (1976) 